Research Article

\title{
The Improved Value-at-Risk for Heteroscedastic Processes and Their Coverage Probability
}

\author{
Khreshna Syuhada 1 \\ Statistics Research Division, Institut Teknologi Bandung, Jalan Ganesa 10, Bandung 40132, Indonesia \\ Correspondence should be addressed to Khreshna Syuhada; khreshna@math.itb.ac.id
}

Received 28 November 2019; Accepted 28 January 2020; Published 10 March 2020

Academic Editor: Zacharias Psaradakis

Copyright (C) 2020 Khreshna Syuhada. This is an open access article distributed under the Creative Commons Attribution License, which permits unrestricted use, distribution, and reproduction in any medium, provided the original work is properly cited.

A risk measure commonly used in financial risk management, namely, Value-at-Risk (VaR), is studied. In particular, we find a $\mathrm{VaR}$ forecast for heteroscedastic processes such that its (conditional) coverage probability is close to the nominal. To do so, we pay attention to the effect of estimator variability such as asymptotic bias and mean square error. Numerical analysis is carried out to illustrate this calculation for the Autoregressive Conditional Heteroscedastic (ARCH) model, an observable volatility type model. In comparison, we find VaR for the latent volatility model i.e., the Stochastic Volatility Autoregressive (SVAR) model. It is found that the effect of estimator variability is significant to obtain $\mathrm{VaR}$ forecast with better coverage. In addition, we may only be able to assess unconditional coverage probability for VaR forecast of the SVAR model. This is due to the fact that the volatility process of the model is unobservable.

\section{Introduction}

Risk management and risk measures have become important topics to discuss among financial and actuarial practitioners as well as academia. They have spent their efforts to seek better risk models and to apply these for real problems. One of the challenging topics in this area is finding a Value-atRisk (VaR) and its assessment of accuracy. VaR may simply be defined as quantile of asset returns' distribution conditional on last observation, e.g., [1]. In fact, $\mathrm{VaR}$ is a value representing tolerated maximum loss of return or portfolio returns; thus, the calculation of $\mathrm{VaR}$ may be multiplied by the market value of portfolio, see [2]. This value is crucial for financial institution for capital reserving. It may also be used as an alarm to avoid the worse financial risk.

From statistical perspective, $\mathrm{VaR}$ forecast is an application of the concept of the prediction limit (upper) for future observations, given a collection of random variables for losses. Suppose that $\left\{Y_{t}\right\}$ is a sequence of random losses with probability distribution determined by the parameter vector $\theta$. The available loss data are $Y_{1}, \ldots, Y_{n}$. In this paper, we are concerned with a common risk measure in financial risk management, namely, Value-at-Risk (VaR). Specifically, we aim at finding $\operatorname{VaR}_{n+1 ; \theta}^{\alpha}$ such that it has coverage (conditional) probability equal to $\alpha$, i.e.,

$$
P_{\theta}\left(Y_{n+1} \leq \operatorname{VaR}_{n+1 ; \theta}^{\alpha} \mid \mathscr{F}_{n}\right)=\alpha, \quad \forall \theta,
$$

where $\mathscr{F}_{n}$ previous information up to time $n$. For known $\theta$, it is straightforward to find a $\mathrm{VaR}_{n+1 ; \theta}^{\alpha}$ that satisfies this condition. In practice, $\theta$ is unknown. An estimative $\operatorname{VaR}_{n+1 ; \widehat{\theta}}^{\alpha}$ forecast is obtained by replacing $\theta$ by an estimator $\widehat{\theta}$. The coverage probability of such $\mathrm{VaR}_{n+1 ; \widehat{\theta}}^{\alpha}$ may not be adequate unless $n$ is very large. It may be shown that the (conditional) coverage probability of $\mathrm{VaR}_{n+1 ; \widehat{\theta}}^{\alpha}$ differs from $\alpha$ by $O\left(n^{-1}\right)$.

We adopt the method of Kabaila and Syuhada [3,4] to modify the estimative $\mathrm{VaR}_{n+1 ; \widehat{\theta}}^{\alpha}$ forecast to obtain an improved ${ }^{+} \mathrm{VaR}_{n+1 ; \widehat{\theta}}^{\alpha}$ forecast with better coverage properties, i.e., it differs from $\alpha$ by $O\left(n^{-3 / 2}\right)$. To find this improved ${ }^{+} \operatorname{VaR}_{n+1}^{\alpha}$ forecast, we need to evaluate the effect of estimator variability such as asymptotic bias and mean square error. When $\hat{\theta}$ is either the maximum likelihood or conditional maximum likelihood estimator, this asymptotic (conditional) bias can be found using the elegant formula described in [5]. 
In this paper, we consider to find estimative and improved VaR forecasts for class of heteroscedastic processes. In particular, we deal with Autoregressive Conditional Heteroscedastic (ARCH) and Stochastic Volatility Autoregressive (SVAR) models. It is well known that the main difference of these two models lies on volatility function; the ARCH model has observable volatility function, whilst in the SVAR model the volatility function remains stochastic or latent. As a consequence, we obtain a $\mathrm{VaR}$ forecast which depends on last observation only for the ARCH model. Besides, the way of finding VaR forecast for the SVAR model is quite difference compared the one in the ARCH model. Furthermore, the estimative and improved VaR forecasts for the SVAR model may only be assessed through unconditional coverage probability.

The remainder of this paper is organized as follows. Section 2 describes the theoretical background of estimative and improved VaR forecasts. Computation of VaR forecast for class of heteroscedastic processes and ARCH and SVAR models are given in Sections 3 and 4, respectively.

\section{Description of the Estimative and Improved VaR Forecast}

Value-at-Risk (VaR) is defined as a maximum loss that can be tolerated at a given level of significance. Let $\mathrm{VaR}_{n+1: \widehat{\theta}}^{\alpha}$ denote the estimative $\mathrm{VaR}$ forecast. The accuracy of ${ }^{n+1 ; i \theta}$ forecast can be calculated through its coverage (conditional) probability:

$$
\left.P_{\theta}\left(Y_{n+1} \leq \operatorname{VaR}_{n+1 ; \widehat{\theta}}^{\alpha}\right) \mid \mathscr{F}_{n}\right)=E_{\theta}\left(F_{\theta}\left(\operatorname{VaR}_{n+1 ; \widehat{\theta}}^{\alpha} \mid \mathscr{F}_{n}\right) \mid \mathscr{F}_{n}\right),
$$

where the expectation is with respect to the conditional distribution of $Y_{n+1}$, given previous information is up to time $n$. To calculate (2), we derive the following Taylor expansion:

$$
\begin{aligned}
F_{\theta}\left(\operatorname{VaR}_{n+1 ; \widehat{\theta}}^{\alpha} \mid \mathscr{F}_{n}\right)= & F_{\theta}\left(\operatorname{VaR}_{n+1 ; \theta}^{\alpha} \mid \mathscr{F}_{n}\right)+\left.\frac{\partial F_{\theta}\left(\operatorname{VaR}_{n+1 ; \theta}^{\alpha} \mid \mathscr{F}_{n}\right)}{\partial \widetilde{\theta}_{r}}\right|_{\widetilde{\theta}_{r}=\theta}\left(\widehat{\theta}_{r}-\theta_{r}\right) \\
& +\left.\frac{1}{2} \frac{\partial^{2} F_{\theta}\left(\operatorname{VaR}_{n+1 ; \tilde{\theta}^{\alpha}}^{\alpha} \mid \mathscr{F}_{n}\right)}{\partial \tilde{\theta}_{r} \partial \widetilde{\theta}_{s}}\right|_{\tilde{\theta}=\theta}\left(\left(\widehat{\theta}_{r}-\theta_{r}\right)\left(\widehat{\theta}_{s}-\theta_{s}\right)\right)+\cdots,
\end{aligned}
$$

where $\widehat{\theta}_{r}$ denotes the $r$ th component of $\widehat{\theta}$ and the Einstein summation notation has been used. It can be seen that the effect of estimator variability contributes significantly to the coverage (conditional) probability of the VaR forecast. As noted by $[5,6]$,

$$
\begin{array}{r}
E_{\theta}\left(\hat{\theta}-\theta \mid \mathscr{F}_{n}\right)=n^{-1} b(\theta)+\cdots, \\
E_{\theta}\left((\widehat{\theta}-\theta)(\widehat{\theta}-\theta)^{T} \mid \mathscr{F}_{n}\right)=i^{-1}(\theta)+\cdots,
\end{array}
$$

where $i(\theta)$ is the expected information matrix. It follows from this that

$$
\left.P_{\theta}\left(Y_{n+1} \leq \operatorname{VaR}_{n+1 ; \widehat{\theta}}^{\alpha}\right) \mid \mathscr{F}_{n}\right)=\alpha+c(\theta) n^{-1}+\cdots
$$

To obtain an improved VaR forecast, we follow the method of Kabaila and Syuhada [3]. Define

$$
\left.d(\theta)=P_{\theta}\left(Y_{n+1} \leq \operatorname{VaR}_{n+1 ; \widehat{\theta}}^{\alpha}\right) \mid \mathscr{F}_{n}\right)-\alpha .
$$

Thus, to order $n^{-1}, d(\theta)=c(\theta)$. The improved VaR forecast, denoted as ${ }^{+} \mathrm{VaR}_{t+1 ; \widehat{\theta}}^{\alpha}$, is

$$
{ }^{+} \operatorname{VaR}_{n+1 ; \widehat{\theta}}^{\alpha}=\operatorname{VaR}_{n+1 ; \widehat{\theta}}^{\alpha}-\frac{d(\widehat{\theta})}{f\left(\operatorname{VaR}_{n+1 ; \widehat{\theta}}^{\alpha} ; \widehat{\theta} \mid \mathscr{F}_{n}\right)},
$$

with coverage (conditional) probability equal to $\alpha+O\left(n^{-3 / 2}\right)$. We begin to find an improved VaR forecast by finding

$$
\left.P_{\theta}\left(Y_{n+1} \leq \operatorname{VaR}_{n+1 ; \widehat{\theta}}^{\alpha}\right) \mid \mathscr{F}_{n}\right),
$$

by simulation. We do this for $\mathrm{ARCH}(1)$ and SVAR(1) processes in the next two sections. Our aim is to illustrate the different ways in obtaining the VaR forecast for these two processes. We may only be able to calculate unconditional coverage probability of the VaR forecast for the SVAR process.

\section{Computation of the Estimative and Improved VaR Forecast for an ARCH(1) Process}

Consider an $\mathrm{ARCH}(1)$ process $\left\{Y_{t}\right\}$ satisfying

$$
\begin{aligned}
Y_{t} & =\sigma_{t} \varepsilon_{t}, \\
\sigma_{t}^{2} & =a_{0}+a_{1} Y_{t-1}^{2},
\end{aligned}
$$

for all integer $t$, where the $\varepsilon_{t}$ are independent and identically $N(0,1)$ distributed and $\theta=\left(a_{0}, a_{1}\right)$. We demonstrate the calculation of the VaR forecast using the simulation algorithm of Kabaila [7] for computing some particular types of conditional expectations.

The estimative VaR forecast is given by

$$
\operatorname{VaR}_{n+1 ; \widehat{\theta}}^{\alpha}=\Phi^{-1}(1-\alpha) \sqrt{\widehat{a}_{0}+\widehat{a}_{1} y_{n}^{2}},
$$

where $\left(\widehat{a}_{0}, \widehat{a}_{1}\right)$ is the conditional maximum likelihood estimator of $\left(a_{0}, a_{1}\right)$. To find the improved VaR forecast, we 
need to find $d(\theta)$, for $\theta=\widehat{\theta}$ and specified $y_{n}$. We do this by first observing that

$$
\begin{aligned}
& P_{\theta}\left(Y_{n+1} \leq \operatorname{VaR}_{n+1 ; \hat{\theta}}^{\alpha} \mid Y_{n}=y_{n}\right) \\
& \quad=P_{\theta}\left(Y_{n+1} \leq \Phi^{-1}(1-\alpha) \sqrt{\widehat{a}_{0}+\widehat{a}_{1} Y_{n}^{2}} \mid Y_{n}=y_{n}\right) \\
& \quad=P_{\theta}\left(\varepsilon_{n+1} \leq \Phi^{-1}(1-\alpha) \sqrt{\frac{\widehat{a}_{0}+\widehat{a}_{1} y_{n}^{2}}{a_{0}+a_{1} y_{n}^{2}}} \mid Y_{n}=y_{n}\right) \\
& \quad=E_{\theta}\left(\Phi\left(\Phi^{-1}(1-\alpha) \sqrt{\frac{\widehat{a}_{0}+\widehat{a}_{1} y_{n}^{2}}{a_{0}+a_{1} y_{n}^{2}}}\right) \mid Y_{n}=y_{n}\right),
\end{aligned}
$$

and estimating this expectation by simulation using the method of Kabaila [7] as follows.

Define $X=\left(Y_{1}, \ldots, Y_{n-1}\right)$ and $Y=Y_{n}$. The conditional expectation (11) is equal to

$$
\vartheta=E_{\theta}(g(X, y) \mid Y=y),
$$

where

$$
g(X, y)=\Phi\left(\Phi^{-1}(1-\alpha) \sqrt{\frac{\widehat{a}_{0}+\widehat{a}_{1} y_{n}^{2}}{a_{0}+a_{1} y_{n}^{2}}}\right),
$$

and $y=y_{n}$. Let $f(y \mid x)$ denote the probability density function of $Y$, conditional on $X=x$, evaluated at $y$. It may be shown that

$$
f(y \mid x)=\phi\left(y_{n} ; \sqrt{a_{0}+a_{1} y_{n}^{2}}\right),
$$

where $\phi(x ; \sigma)$ denotes the probability density function of $X \sim N\left(0, \sigma^{2}\right)$, evaluated at $x$.

The simulation used to estimate $\vartheta$ consists of $M$ independent simulation runs, where the $k$ th run consists of the following steps:

Step 1: simulate an observation $x^{k}$ of $X=\left(Y_{1}, \ldots\right.$, $\left.Y_{n-1}\right)$

Step 2: calculate and store $f\left(y \mid x^{k}\right)$ and $g\left(x^{k}, y\right)$

The estimate of $\vartheta$ is

$$
\frac{\sum_{k=1}^{M} f\left(y \mid x^{k}\right) g\left(x^{k}, y\right)}{\sum_{\ell=1}^{M} f\left(y \mid x^{\ell}\right)} .
$$

The standard error of this estimate is found using Theorem 4.2 of Kabaila [7].

We carry out simulation to illustrate computation of the estimative and improved VaR forecasts. We take the following case: $\widehat{\theta}=\left(\widehat{a}_{0}, \widehat{a}_{1}\right)=(0.15,0.9), n=200$, and $y_{n}=0.1$. The estimative $0.95 \mathrm{VaR}$ forecast $\operatorname{VaR}_{n+1: \widehat{\theta}}^{0.95}=0.6861$. The conditional coverage probability of estimative $\mathrm{VaR}$ forecast is calculated by using $M=10,000$ simulation runs. The resulting estimate of (11) is 0.9423 , with standard error 0.00019 . The improved $0.95 \mathrm{VaR}$ forecast ${ }^{+} \mathrm{VaR}_{n+1: \widehat{\theta}}^{0.95}$ is therefore estimated to be 0.7173 . The standard error of the estimate of (11) indicates that this estimate leads to a sufficiently accurate estimate of the improved VaR forecast. Note that computation is performed using MATLAB and the MATLAB statistics toolbox.

\section{VaR Forecast and Its Coverage Probability of a SVAR(1) Process}

An alternative process for modeling volatility is Stochastic Volatility Autoregressive (SVAR) process. Unlike ARCH, the SVAR process has latent volatility function. Nonetheless, the SVAR(1) model is a good representation, from theoretical viewpoint, of the behavior of the returns in the real financial markets.

The purpose of this section is to find estimative and improved VaR forecast for such SVAR process along with their coverage probability and, most importantly, to show how different in finding VaR forecast for SVAR model in comparison to the one of ARCH model. The desirability of having coverage probability $\alpha$ for the VaR forecast of the SVAR process has not been discussed by many authors. Most of the papers on the SVAR have investigated the parameter estimation method, estimating volatility and/or evaluating volatility prediction in the context of meansquared-error of forecasting.

We consider the SVAR(1) model which is developed as follows. Suppose that $Y_{t}$ is an asset return at time $t$, and we assume that the average return is zero. The distribution of $Y_{t}$, conditional on the variance, is normal with mean zero and variance $\exp \left(V_{t}\right)$, where $V_{t}$ follows an autoregressive process of order one or $\mathrm{AR}(1)$ process. In other words,

$$
\begin{aligned}
& Y_{t}=\exp \left(\frac{V_{t}}{2}\right) \varepsilon_{t}, \\
& V_{t}=\gamma+\delta V_{t-1}+\eta_{t},
\end{aligned}
$$

for $t=0,1, \ldots$, where the $\varepsilon_{t} s$ are independent and identically distributed (i.i.d.) $N(0,1)$ and $\eta_{t}$ 's are i.i.d. $N\left(0, \sigma_{\eta}^{2}\right)$. The arrays of $\eta_{t}$ 's and $\varepsilon_{t}$ 's are independent. Let $\theta=\left(\gamma, \delta, \sigma_{\eta}^{2}\right)$ be the parameter of the SVAR(1) model, where $\delta$ is the persistence parameter, whilst $\sigma_{\eta}^{2}$ denotes the volatility of the volatility shock. Here, we restrict to the case that the SVAR(1) model is covariance stationary, i.e., the persistence parameter $|\delta|<1$. The assumption of normality for $\varepsilon_{t}$ can be relaxed by using other distributions, see, for example, [8] who has shown the dominance of SVAR with heavy-tailed distributions compared to SVAR-normal. When the Gaussian distribution is employed for both $\varepsilon_{t}$ and $\eta_{t}$, we call the model as a "Gaussian SVAR(1) model."

We begin to find the $\mathrm{VaR}$ forecast as follows. Consider the case of a stationary Gaussian SVAR(1) model, i.e., the persistence parameter $\delta<1$ and $\varepsilon_{t}$ and $\eta_{t}$ are $N(0,1)$ and $N\left(0, \sigma_{\eta}^{2}\right)$ distributed, respectively. The unconditional distribution of $V_{t}$ is $N\left(\mu_{V}, \sigma_{V}^{2}\right)$, where $\mu_{V}=\gamma /(1-\delta) o$ and $\sigma_{V}^{2}=\sigma_{\eta}^{2} /\left(1-\delta^{2}\right)$. We observe that 


$$
\begin{aligned}
P_{\theta}\left(Y_{n+1} \leq z\right) & =P_{\theta}\left(\exp \left(\frac{V_{n+1}}{2}\right) \varepsilon_{n+1} \leq z\right) \\
& =E_{\theta}\left(P_{\theta}\left(\exp \left(\frac{V_{n+1}}{2}\right) \varepsilon_{n+1} \leq z \mid V_{n+1}\right)\right) \\
& =E_{\theta}\left(\Phi\left(\frac{z}{\exp \left(V_{n+1} / 2\right)}\right)\right) \\
& =\int_{-\infty}^{\infty} \Phi\left(\frac{z}{\exp (v / 2)}\right) f_{V}(v ; \theta) \mathrm{d} v
\end{aligned}
$$

where $\Phi(\cdot)$ is the cumulative distribution function (cdf) of the standard normal distribution and $f_{V}(\cdot ; \theta)$ denotes the unconditional probability density function (pdf) of $V$.

Define

$$
H(z, \theta, \alpha)=\int_{-\infty}^{\infty} \Phi\left(\frac{z}{\exp (v / 2)}\right) f_{V}(v ; \theta) \mathrm{d} v-\alpha,
$$

and also define $\operatorname{VaR}_{n+1 ; \theta}^{\alpha}$ to be the solution for $z$ of $P_{\theta}\left(Y_{n+1} \leq z\right)=\alpha$, i.e.,

$$
H(z, \theta, \alpha)=0 .
$$

For a specified estimator $\widehat{\theta}$, we will obtain $z=\operatorname{VaR}_{n+1 ; \widehat{\theta}}^{\alpha}$, which is the "estimative VaR forecast."
The evaluation of the integral in the definition of $H(z, \theta, \alpha) o$ analytically is not straightforward. Nonetheless, we can evaluate this numerically as follows. Firstly, we carry out a truncation of this integral. The lower and upper bound are set to be $\mu_{V}-k \sigma_{V}$ and $\mu_{V}+k \sigma_{V}$, respectively, where $k$ is a positive integer. Thus, the error of truncation is bounded above by

$$
\begin{aligned}
& \int_{-\infty}^{\mu_{V}-k \sigma_{V}} \Phi\left(\frac{z}{\exp (v / 2)}\right) f(v ; \theta) \mathrm{d} v \\
& \quad+\int_{\mu_{V}+k \sigma_{V}}^{\infty} \Phi\left(\frac{z}{\exp (v / 2)}\right) f(v ; \theta) \mathrm{d} v \\
& \quad \leq 2 \int_{\mu_{V}+k \sigma_{V}}^{\infty} f(v ; \theta) \mathrm{d} v=2(1-\Phi(k)) .
\end{aligned}
$$

If we set $k=4$, for instance, then the truncation error is bounded above by $6.33 \times 10^{-5}$. Alternatively, if we truncate the integral with lower bound $\mu_{V}-5 \sigma_{V}$ and upper bound $\mu_{V}-5 \sigma_{V}$, i.e., $k=5$, then we obtain the truncation error bounded above by $5.733 \times 10^{-7}$. The second step is conducting a numerical integration and find $\mathrm{VaR}_{n+1 ; \theta}^{\alpha}$ by numerical solution of $H(z, \theta, \alpha)=0$.

Now, in order to compute the improved VaR forecast, we first observe that

$$
\begin{aligned}
P_{\theta}\left(Y_{n+1} \leq \operatorname{VaR}_{n+1 ; \widehat{\theta}}^{\alpha}\right) & =P_{\theta}\left(\varepsilon_{n+1} \leq \frac{\operatorname{VaR}_{n+1 ; \widehat{\theta}}^{\alpha}}{\exp \left(V_{n+1} / 2\right)}\right) \\
& =E_{\theta}\left(P_{\theta}\left(\varepsilon_{n+1} \leq \frac{\mathrm{VaR}_{n+1 ; \widehat{\theta}}^{\alpha}}{\exp \left(V_{n+1} / 2\right)} \mid Y_{1}, \ldots, Y_{n}, V_{n+1}\right)\right) \\
& =E_{\theta}\left(\Phi\left(\frac{\left.\mathrm{VaR}_{n+1 ; \widehat{\theta}}^{\alpha}\right)}{\exp \left(V_{n+1} / 2\right)}\right)\right),
\end{aligned}
$$

and, as before, we estimate this (unconditional) expectation by simulation.

Define

$$
d(\theta)=P_{\theta}\left(Y_{n+1} \leq \operatorname{VaR}_{n+1 ; \widehat{\theta}}^{\alpha}\right)-\alpha
$$

and let $f(\cdot ; \theta)$ be the probability density function of $Y_{n+1}$. The improved $\mathrm{VaR}$ forecast ${ }^{+} \mathrm{VaR}_{n+1 ; \widehat{\theta}}^{\alpha}$ is given by

$$
{ }^{+} \operatorname{VaR}_{n+1 ; \widehat{\theta}}^{\alpha}=\operatorname{VaR}_{n+1 ; \widehat{\theta}}^{\alpha}-\frac{d(\widehat{\theta})}{f\left(\operatorname{VaR}_{n+1 ; \widehat{\theta}}^{\alpha} ; \widehat{\theta}\right)} .
$$

Monte Carlo simulation estimates to obtain the estimative and improved VaR forecasts are obtained as follows. We begin by running simulation data from a stationary Gaussian SVAR(1) model with sample size $n=100$ and parameter $\theta=(0.01,0.95,0.17)$. The parameter estimation is conducted by applying the Maximum Likelihood-Efficient
Important Sampling (ML-EIS) method, and we have used $m=50$ simulation runs to obtain $\widehat{\theta}$.

For a single run of the simulation described above, we obtain $\widehat{\theta}=(0.016,0.962,0.178)$. The corresponding estimative $0.95 \mathrm{VaR}$ forecast $\mathrm{VaR}_{n+1 ; \widehat{\theta}}^{0.95}$ is obtained by evaluating $H(z, \widehat{\theta}, 0.95)$ via numerical integration in which the $\operatorname{VaR}_{n+1 ; \hat{\theta}}^{\alpha}$ is computed by numerical solution of $H(z, \widehat{\theta}, 0.95)=0$ for $z$. The resulting estimative $0.95 \mathrm{VaR}$ forecast $\mathrm{VaR}_{n+1 ; \widehat{\theta}}^{0.95}$ is 2.28 . Note that when evaluating the integral we have chosen $k=4$ so that the truncated integral has error bounded above by $6.33 \times 10^{-5}$, which is reasonably small.

We find the improved $\mathrm{VaR}$ forecast that corresponds to this estimative one by using $\hat{\theta}$ as the "true parameter value" in the simulation. We carry out $M=1000$ simulation runs. These simulations are used to estimate $d(\widehat{\theta})$ in the obvious way. The improved $0.95 \mathrm{VaR}$ forecast ${ }^{+} \mathrm{VaR}_{n+1 ; \widehat{\theta}}^{0.95}=2.511$, which differs significantly from the estimative $0.95 \mathrm{VaR}$ forecast $\mathrm{VaR}_{n+1 ; \hat{\theta}^{0}}^{0.95}$. 
TABle 1: The VaR forecast (and improvement) for Generalized ARCH and SVAR models and some extensions of volatility models.

\begin{tabular}{lcc}
\hline Volatility models & $\operatorname{VaR}_{n+1 ; \widehat{\theta}}^{0.99}$ & $\operatorname{VaR}_{n+1 ; \widehat{\theta}}^{0.95}$ \\
\hline GARCH- $n(1,1)$ & $0.0210(0.0274)$ & $0.0148(0.0218)$ \\
GARCH- $t(1,1)$ & $0.0236(0.0289)$ & $0.0167(0.0229)$ \\
SVAR- $n(1)$ & $0.0240(0.0330)$ & $0.0200(0.0258)$ \\
SVAR- $t(1)$ & $0.0245(0.0311)$ & $0.0216(0.0277)$ \\
GJR-GARCH- $n(1,1)$ & $0.0228(0.0341)$ & $0.0162(0.0268)$ \\
GJR-GARCH- $t(1,1)$ & $0.0249(0.0367)$ & $0.0176(0.0278)$ \\
TGARCH- $n(1,1)$ & $0.0228(0.0266)$ & $0.0195(0.0217)$ \\
TGARCH- $t(1,1)$ & $0.0243(0.0299)$ & $0.0162(0.0265)$ \\
\hline
\end{tabular}

4.1. Numerical Illustration. In order to describe clearly in real application setting for the abovementioned VaR forecast methods, we provide a numerical study of VaR forecasts especially for the case Generalized ARCH and SVAR models (and some extensions of volatility models i.e., GJR Generalized ARCH and Threshold Generalized ARCH) with some distributions assumptions. We have used NASDAQ and NYSE data (YahooFinance) for duration of 1 January 2007 till 10 December 2016 that comprises of 2516 observations. The choice of volatility models may not be easy with regard to different results as the effect of involving features attached to the models (Table 1).

\section{Concluding Remark}

The computation of estimative and improved VaRs may be extended in two different directions. First, as pointed out by Dendramis et al. [9] and So and Yu [10], e.g., it is applied to volatility models with heavy-tailed distributions, such as $t$ and GED, as well as models to capture leverage effect and asymmetric, such as Exponential Generalized ARCH or GJR Generalized ARCH. The second direction is by taking the adjusted VaR forecast that ensures or satisfies the coherent property. Some possible risk measures include modification of mean for observations beyond $\mathrm{VaR}$ or involving other dependent observations.

\section{Data Availability}

NASDAQ and NYSE data taken from Yahoo Finance were used to support the findings of this study.

\section{Disclosure}

This paper has been presented in the 9th International Triennial Calcutta Symposium on Probability and Statistics, 2015.

\section{Conflicts of Interest}

The authors declare that they have no conflicts of interest.

\section{Acknowledgments}

The author is indebted to Prof. Paul Kabaila (La Trobe University) for thoughtful discussion. Financial support of "Riset ITB" from Institut Teknologi Bandung is acknowledged. The author would also like to thank Evi Lestari for assistance in real data computation.

\section{References}

[1] P. Christoffersen and D. Pelletier, "Backtesting value-at-risk: a duration-based approach," Journal of Financial Econometrics, vol. 2, no. 1, pp. 84-108, 2004.

[2] M. Wong, W. Cheng, and C. Wong, "Market risk management of banks: implication from the accuracy of Value-at-Risk forecasts," Journal of Forecasting, vol. 22, no. 1, pp. 23-33, 2003.

[3] P. Kabaila and K. Syuhada, "Improved prediction limits for $\operatorname{AR}(p)$ and $\operatorname{ARCH}(p)$ processes," Journal of Time Series Analysis, vol. 29, no. 2, pp. 213-223, 2008.

[4] P. Kabaila and K. Syuhada, "The asymptotic efficiency of improved prediction intervals," Statistics and Probability Letters, vol. 80, no. 17-18, pp. 1348-1353, 2010.

[5] P. Vidoni, "Improved prediction intervals for stochastic process models," Journal of Time Series Analysis, vol. 25, no. 1, pp. 137-154, 2004.

[6] O. E. Barndorff-Nielsen and D. R. Cox, "Prediction and asymptotics," Bernoulli, vol. 2, no. 4, pp. 319-340, 1996.

[7] P. Kabaila, "An efficient simulation method for the computation of a class of conditional expectations," Australian \& New Zealand Journal of Statistics, vol. 41, no. 3, pp. 331-336, 1999.

[8] K. Syuhada, Neo Normal Stochastic Volatility Models, Unpublished M.Sc thesis, Curtin University, Perth, Australia, 2004.

[9] Y. Dendramis, G. E. Spungin, and E. Tzavalis, "Forecasting VaR models under different volatility processes and distributions of return innovations," Journal of Forecasting, vol. 33, no. 7, pp. 515-531, 2014.

[10] M. K. P. So and P. L. H. Yu, "Empirical analysis of GARCH models in value at risk estimation," Journal of International Financial Markets, Institutions \& Money, vol. 16, no. 2, pp. 180-197, 2006. 